\title{
Streamlining Asset Maintenance throughout Analysis of its Usage Data
}

\author{
Hong-Bae Jun, Maurice Ruibal, Dimitris Kiritsis, and Paul Xirouchakis \\ EPFL (STI-IPR-LICP), Staion 9, ME B1, \\ CH-1015 Lausanne, Switzerland \\ WWW home page: http://licpwww.epfl.ch
}

\begin{abstract}
Recently, with the advent of emerging technologies such as radio frequency identification (RFID), various sensors, and wireless telecommunication, we can have the visibility of asset status information over the whole asset lifecycle. It gives us new challenging issues for improving the efficiency of asset operations. One of the most challenging problems is the predictive maintenance that makes a prognosis of the asset status via a remote monitoring, predicts the asset's abnormality, and executes suitable maintenance actions such as repair and replacement. In this study, we will develop a prognostic decision algorithm to take suitable maintenance actions by analyzing the degradation status of an asset. To evaluate the proposed approach, we carry out a case study for a heavy machinery.
\end{abstract}

\section{Introduction}

In general, asset maintenance is defined as all technical and managerial actions taken during usage period to maintain or restore the required functionality of an asset. Maintenance has mainly three types: breakdown maintenance (corrective maintenance), preventive maintenance, and predictive maintenance. Among them, the predictive maintenance focuses on the prediction of degradation process of the asset, which is based on the assumption that most abnormalities do not occur instantaneously, and usually there are some kinds of degradation process from normal states to abnormalities [1]. Hence, unlike breakdown maintenance and preventive maintenance, the predictive maintenance concentrates on degradation monitoring and prognostics rather than fault detection and diagnostics of components. It enables us to identify and solve problems in advance before asset damage occurs. Until now, it is difficult to achieve effectiveness of maintenance operations because there is no information visibility during asset usage period. Although there are recommendations concerning maintenance strategy from vendors or manufacturers, they are neither practical nor cost-effective because they are too

Please use the following format when citing this chapter:

Jun, H.-B., Ruibal, M., Kiritsis, D. and Xirouchakis, P., 2008, in IFIP International Federation for Information Processing, Volume 257, Lean Business Systems and Beyond, Tomasz Koch, ed.; (Boston: Springer), pp. 111-119. 
generic. However, recently, with emerging technologies such as radio frequency identification (RFID), various sensors, micro-electro-mechanical system (MEMS), and wireless tele-communication, asset embedded information devices such as RFID tags are expected to be rapidly used for gathering and monitoring the status data of assets during their usage period. Under the new environment, an asset embedded information device can $\log$ the asset history related to distributing route, usage conditions, failure, maintenance or service events, and so on. Therefore, using this information gives us new challenging issues for improving the efficiency of asset maintenance operations. We can make a prognosis of asset status, predict asset's abnormality, and execute proactive maintenance, upgrade or decommission, i.e. do predictive maintenance.

To implement a predictive maintenance approach, it is required to resolve several research issues related to data gathering, analyzing, decision, and actions. Among them, in this study, we focus on the analyzing and decision making levels. For this, we will develop an algorithm that can analyze the degradation status and determine the prognostic maintenance strategy. If a degradation status can be measured and detected in a real time way, then predictive maintenance activities can be performed before a worse degradation or failure occurs. In other words, maintenance interval can be optimized through predictive maintenance. It means that we can reduce nonnecessary maintenance cost. Hence, it is important to develop a proactive decision algorithm for predicting the time of product's abnormality; and executing the appropriate maintenance actions. It will minimize the overall maintenance cost. To evaluate the proposed approach, we carry out a case study for a heavy machinery.

The rest of the paper is organized as follows: In section 2, we look into relevant previous research. In section 3 , we address a predictive maintenance algorithm. In section 4 , we introduce a case study.

\section{Previous Research}

There have been several research works about predictive maintenance. For example, $\mathrm{Fu}$ et al. [1] proposed a predictive maintenance framework for hydroelectric generating unit. They presented three key elements for the predictive maintenance such as monitoring and forecasting, diagnosis and prognosis, and decision-making. In addition, Bansal et al. [2] described a real-time predictive maintenance system for machine systems. The aim of the proposed system is to localize and detect abnormal electrical conditions in order to predict mechanical abnormalities that indicate, or may lead to the failure of a motor. They used a neural network approach to predict parameters of a machine. On the other hand, the predictive maintenance has been highlighted with the concept of e-maintenance and the product identification technology. For example, Koç and Lee [3] addressed the concept of web-enabled predictive maintenance in an intelligent e-maintenance system which is implemented via Internet and showed its system elements. Lee [4] introduced a new methodology of predictive maintenance, called machinery dynamics and data fusion through remote machinery monitoring. They also presented an example of a remote wireless application currently in use for monitoring machinery in industrial plants. In 
addition, recently, Djurdjanovic et al. [5] proposed the framework of watchdog agent for predictive condition-based maintenance by realizing multi-sensor assessment and prediction of machine or process performance. The concept of watchdog agent based its degradation assessment on the readings from multiple sensors that measure critical properties of the process or machinery under a networked and tether-free environment. Although there have been some relevant research works so far, they have some limitations from the following viewpoints. First, there is a lack of research to address the issue of combining information technologies with the predictive maintenance. Another limitation is that the predictive maintenance is still an undeveloped area and its approaches are unreliable. Hence, the current approaches have the limitation in detailed methods or validated predictive models.

\section{Predictive Maintenance Approach}

To cope with the previous limitations, in this study, we develop a prognostic decision algorithm to select the best maintenance strategy throughout the analysis of asset usage data. The following are notations used in this study.

\section{Notations}

$T_{D} \quad$ Designed life time

$T_{M} \quad$ Asset operation time

$T^{*} \quad$ Remaining life time

$T^{\prime} \quad$ Theoretical remaining life time, $T^{\prime}=T_{D}-T_{M}$

$\varepsilon \quad$ Arbitrary small number empirically determined by companies

The following is the detailed procedure for a predictive maintenance approach.

\section{Predictive maintenance algorithm}

Step 1. Collect status data of an asset

In this step, using an embedded information device, we gather product status data. Here, the embedded information device plays a role in sensing and gathering the asset status data, and transmitting them to a relevant information system. Depending on the type of the asset, various kinds of embedded information devices can be used, e.g. RFID tag, on-board computer, and so on.

Step 2. Estimate remaining life time of the asset at a certain time $T$

With gathered data, we can estimate the remaining life time of the asset. For this, a prediction model for calculating the remaining life time of the asset should be developed considering the asset's characteristics.

Step 3. Check asset status

With estimated remaining life time, check whether the asset can last as long as it was originally intended to. If $T^{*} \leq T^{\prime}-\varepsilon$, do predictive maintenance. Here, $T$ will be the best time to take maintenance actions. Else if $T^{*} \geq T^{\prime}+\varepsilon$, then take no actions, or adjust the severity of mission profile for the use of more intensive 
applications, or inform design department that the asset is overdimensioned designed. Otherwise, let the asset be without taking any maintenance actions.

Step 4. Decide suitable maintenance strategy

1. Generate possible maintenance strategies considering what to do, when to do, how to do, and where to do.

2. For each strategy, build up a maintenance cost model

3. Calculate the maintenance cost for each maintenance strategy

4. Compare them and select the best one

Step 5. Implement the selected maintenance strategy

Following the selected maintenance strategy, take suitable maintenance actions.

\section{Case study}

To evaluate our approach, we carry out a case study for a heavy machinery of $A A A$ company in France. The $A A A$ company uses an embedded information device (onboard computer) with sensors in a structural part of the heavy machinery to gather its usage data. With gathered data, we analyze the degradation of the structural part; predict remaining life time, and decide the best cost effective maintenance operation, which are described below in detail. The following are notations used in the case study.

\section{Notations}

$a \quad$ Crack length coefficient (depending on the crack geometry)

$a_{i} \quad$ Crack length coefficient at a certain measurement time

$a_{f} \quad$ Critical crack length coefficient

$\dot{a} \quad$ Crack growth rate

$C \quad$ Empirical parameter

$C_{B} \quad$ Logistics cost of borrowed machinery (transport to customer)

$C_{C} \quad$ Cost of compensation for customer

$C_{D} \quad$ Disassembly cost

$C_{L_{i}} \quad$ Labor cost for repair or replace, $\mathrm{i}=1,2$ (1: repair, 2: replace)

$C_{P_{i}} \quad$ Part cost for repair or replace, $\mathrm{i}=1,2$ (1: repair, 2 : replace)

$C_{P_{3}} \quad$ New part or component cost

$C_{T} \quad$ Transportation cost for delivering a failed machine to a dealer or transporting a maintenance engineer to a customer site

$c_{R} \quad$ Repair consumable hourly cost

$\mathrm{F} \quad$ The number of stress cycles

$h_{R} \quad$ Hourly labor cost

$i_{R} \quad$ Hourly immobilization cost 


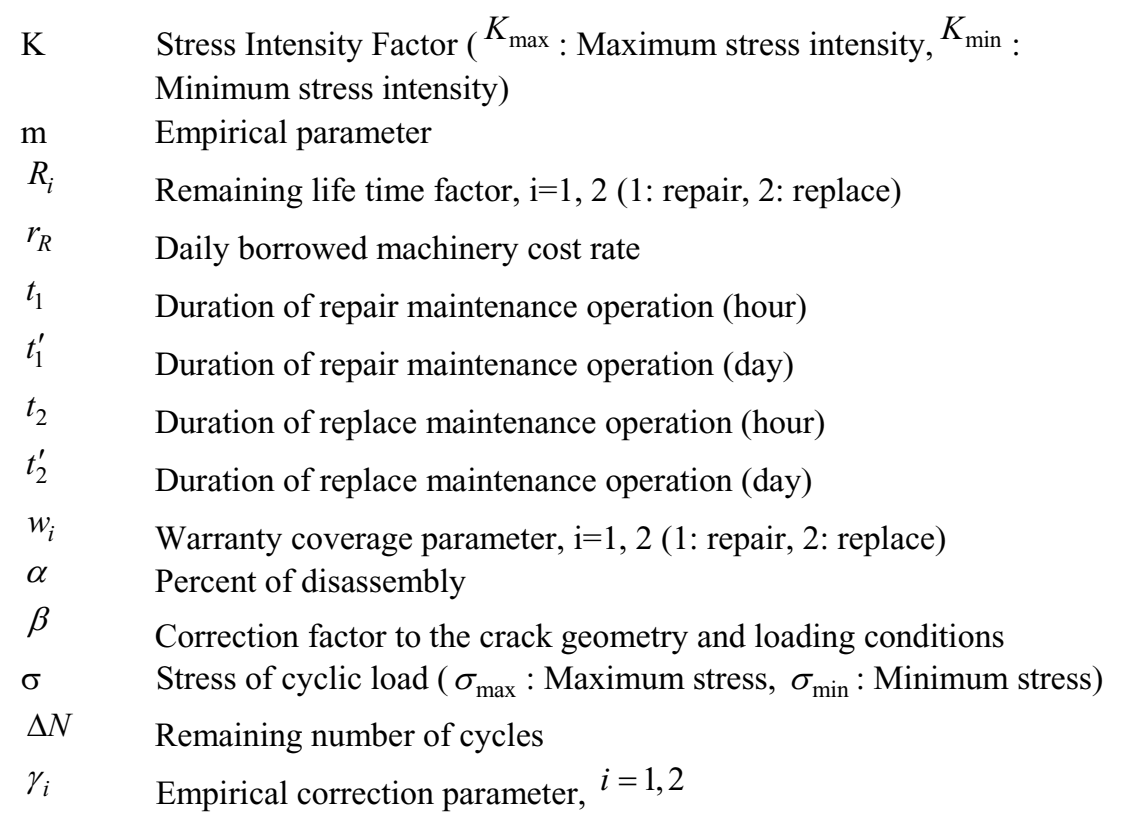

Step 1. Collect usage status data of the heavy machinery at $T$

We can gather machine operating time $\left(T_{M}\right)$, stress levels $(\Delta \sigma)$ of a structural part, the number of stress cycles $(F)$, and crack propagation data $(a, \dot{a})$ from an embedded information device with sensors.

Step 2. Estimate remaining life time of the structural part of the heavy machinery at a certain time $T$. The following is a detailed procedure as to how to estimate the remaining life time of the structural part.

1. Find mission profile parameters $(\Delta \sigma, F)$

Considering the future usage of the structural part, select suitable $\Delta \sigma$ and $F$ in the user model.

2. Calculate stress intensity factor $(\Delta K)$

With the following equation, we calculate stress intensity factor. Here $\beta$ can be empirically determined from previous experience.

$$
\begin{gathered}
\Delta K=K_{\max }-K_{\min }=\beta \cdot \Delta \sigma \cdot \sqrt{\pi \cdot a} \text { where } K_{\max }=\beta \cdot \sigma_{\max } \cdot \sqrt{\pi \cdot a} \text { and } \\
K_{\min }=\beta \cdot \sigma_{\min } \cdot \sqrt{\pi \cdot a}
\end{gathered}
$$

3. Select suitable $C$ and $m$ at Paris-Erdogan model [6] (eq. (1))

Using fracture mechanics theory [6], we can know the relation between crack propagation speed $(\dot{a})$ and stress intensity factor $(K)$. The law relating crack propagation speed $\dot{a}$ to $\Delta K$ is derived from experimental results. The most widely accepted one for several materials is the Paris-Erdogan model.

$$
\dot{a} \cong \frac{d a}{d N}=C(\Delta K)^{m}
$$


where $C$ and $m$ are empirical parameters determined by the fitting equation (1) to the fatigue data. Factors which affect crack propagation can be grouped into the following categories: material microstructure, processing, load spectrum, environment and geometry of a component. In this study, we will only consider load spectrum, component geometry, and working environment for the crack propagation modeling and remaining life time prediction.

4. Calculate the remaining life time $\left(T^{*}\right)$

Using the following equation, we can calculate the remaining life time of the structural part.

$$
T^{*}=\frac{\Delta N}{F} \text { where } \Delta N=\int_{a_{i}}^{a_{f}} \frac{1}{C \cdot(\Delta K)^{m}} d a=\int_{a_{i}}^{a_{f}} \frac{1}{C \cdot(\beta \cdot \Delta \sigma)^{m} \cdot(\pi \cdot a)^{m-2}} d a
$$

Step 3. Check the status of the structural part

Let us take a simple example. If we get $T^{*}=7500$ under $T_{D}=11000$, $T_{M}=2294$, and $\varepsilon=48$, then, since $T^{*} \leq T^{\prime}-\varepsilon$, we can conclude 'do predictive maintenance operation'. Here, $T\left(=T_{M}\right)$ is the best time to take the maintenance operation.

Step 4. Decide suitable maintenance strategy

Figure 1 shows the possible maintenance strategies depending on the variation of maintenance policy, amount of disassembly, and maintenance location. First, we should decide what to do: repair or replace. Here, repair indicates maintenance operations that all original components of the structural part remain and new material is added. On the other hand, replace means the operation that substitution of some components of the structural part with possibly new material being added. Second, we should decide how to perform the selected maintenance operation with respect to disassembly: no, partial, and total disassembly. Finally, we should decide where to do maintenance operation: on site or at dealer site. Depending on them, we can generate 14 maintenance strategies as depicted in Figure 1.

In this study, to show a simple example of our approach, we consider a failure of a component of the structural part: a damaged component $L$. The following equations are two maintenance cost models, $A, B$ (see dotted lines in Figure 1).

A: Repair cost function

$$
\begin{aligned}
& w_{1} \cdot R_{1} \cdot \gamma_{1}\left(C_{L_{1}}+C_{P_{1}}+C_{C}+C_{T}\right)=\left(\frac{T_{D}-T_{M}}{T_{D}}\right)\left(1-\frac{T^{*}}{T_{D}-T_{M}}\right) \cdot \gamma_{1} . \\
& \left(\left(h_{R} t_{1}+\alpha C_{D}\right)+\left(C_{R} t_{1}\right)+\left(C_{B}+r_{R} t_{1}^{\prime}+i_{R} t_{1}\right)+C_{T}\right)
\end{aligned}
$$

\section{B: Replace cost function}

$$
\begin{gathered}
w_{2} \cdot R_{2} \cdot \gamma_{2}\left(C_{L_{2}}+C_{P_{2}}+C_{C}+C_{T}\right)=\left(1-\frac{T_{D}-T_{M}}{T_{D}}\right)\left(\frac{T^{*}}{T_{D}-T_{M}}\right) \\
\left(\left(h_{R} \cdot t_{2}+\alpha C_{D}\right)+\left(C_{P_{3}}+C_{R} \cdot t_{2}\right)+\left(C_{B}+r_{R} t_{2}^{\prime}+i_{R} t_{2}\right)+C_{T}\right)
\end{gathered}
$$


In the repair and replace functions, the warranty coverage $\left(w_{i}\right)$ is an important factor since it is an element which indicates to the manufacturer of the system what it will cost him in order to maintain a machine still covered by warranty. Furthermore, the remaining life time factor $\left(R_{i}\right)$ is also important to decide the repair or replace option. As the remaining life time factor becomes increasing and the warranty coverage becomes reduced, the repair option is more preferable to the replace option. To take it into account, we consider $w_{1}, w_{2}, R_{1}, R_{2}$ in the functions. The labor cost contains the direct labor costs of maintenance personnel during repair/replace operation period and indirect labor costs considering the percentage of disassembly. The compensation cost indicates the cost related to the compensation given to the customer while his machine is in repair/replace, e.g. cost related to lending him another machine. In addition, $\gamma_{1}$ and $\gamma_{2}$ should be empirically determined by companies. Here, $\gamma_{1}$ and $\gamma_{2}$ were estimated from relevant failure reports of the $C$ company that contain real repair and replacement costs. The values of other parameters could be obtained from the company. The following are calculations of repair cost and replace cost.

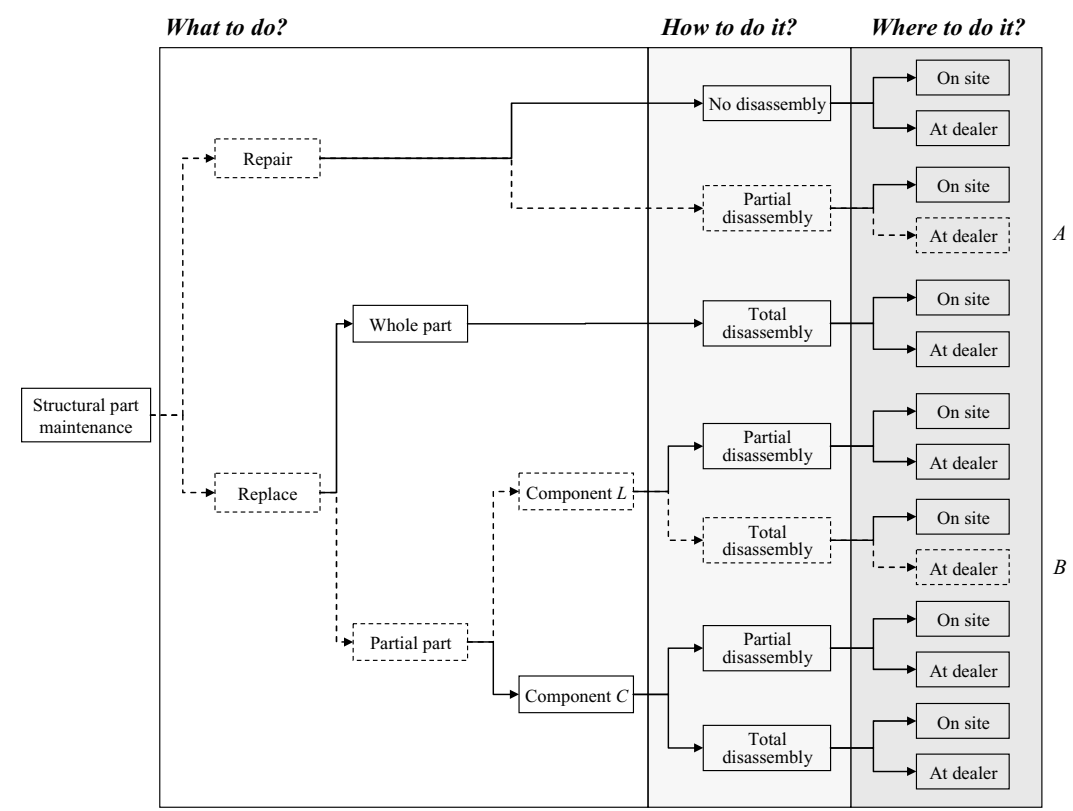

Fig. 1. Maintenance strategies for the structural part

A: Repair cost

$$
\begin{aligned}
& \left(\frac{11000-2294}{11000}\right)\left(1-\frac{7500}{11000}\right) \cdot 0.40979 \\
& ((100 \cdot 3+0.1 \cdot 200)+(30 \cdot 3)+(1000+500 \cdot 1)+1000)=300.30
\end{aligned}
$$


where $T_{D}=11000, \quad T_{M}=2294, \quad t_{1}=3, \quad t_{1}^{\prime}=1, \quad \alpha=10 \%, \quad \gamma_{1}=0.40979$, $C_{D}=200 \$, C_{R}=30, C_{B}=1000, C_{T}=1000, h_{R}=100, r_{R}=500$ and $i_{R}=0$.

B: Replace cost

$$
\begin{aligned}
& \left(1-\frac{11000-2294}{11000}\right)\left(\frac{7500}{11000}\right) \cdot 1.11276 \\
& ((100 \cdot 8+1 \cdot 200)+(3000+30 \cdot 8)+(1000+500 \cdot 1)+1000)=1066.42
\end{aligned}
$$

where $\alpha=100 \%, \gamma_{1}=1.11276, t_{2}=8, t_{2}^{\prime}=1, C_{P_{3}}=3000, C_{R}=30, C_{B}=1000$, and $r_{R}=500$. As you can see, the repair cost for the damaged component $L$ is cheaper than that of replace option. Hence, repairing the component with partial disassembly at the dealer site is preferable to replacing the whole component.

Step 5. Implement the selected maintenance strategy

Since the repair option is more economic than the replace option, we take repair maintenance operation in the dealer site at 7500 operation hours.

Throughout the case study, we could see that our decision algorithm provides us with a practical guide on which type of maintenance operation (repair), when (7500 operation hours), where (at the dealer site), and how it should perform (partial disassembly) based on degradation analysis of the structural part.

\section{Conclusion}

In this study, we have dealt with predictive asset maintenance. We have addressed how to estimate the remaining life time of assets based on gathered asset status data. Furthermore, we have provided a cost based decision support method for selecting the best predictive maintenance operation. As a case study, we have developed a performance estimation method for a structural part of a heavy machinery during its usage period considering usage status data. We have also combined it with the selection of the best maintenance operation strategy. Main contribution of our study is to show how asset usage data gathered by information technologies can be used for streamlining maintenance operations in a proactive way.

\section{Acknowledgements}

The work reported in this paper was based on the PROMISE project that is currently under development (www.promise.no). Therefore, we wish to express our deep gratitude to all PROMISE partners. Specially thanks to Cecile Corcelle. 


\section{Reference}

1. C. Fu, L. Ye, Y. Liu, R. Yu, B. Iung, Y. Cheng, and Y. Zeng, Predictive maintenance in intelligent-control-maintenance-management system for hydroelectroic generating unit, IEEE Transactions on energy conversion, 19(1), 179186 (2004).

2. D. Bansal, D. J. Evans, and B. Jones, A real-time predictive maintenance system for machine systems, International Journal of Machine Tools and Manufacture, 44, 759-766 (2004).

3. M. Koç and J. Lee, A system framework for next-generation E-maintenance systems, Transaction of Chinese Mechanical Engineer, 12 (2001).

4. L. D. Lee, Using wireless technology and the Internet for predictive maintenance, Hydrocarbon processing, 80(5), 77-96 (2001).

5. D. Djurdjanovic, J. Lee, and J. Ni, Watchdog Agent-an infotronics-based prognostics approach for product performance degradation assessment and prediction, Advanced Engineering Informatics, 17, 109-125 (2003).

6. N. E. Dowling, Mechanical behavior of materials (Prentice hall, 1999). 